\title{
SUCCESSFUL USE OF CRUSHED FORMULATION OF DABRAFENIB AND TRAMETINIB IN A PEDIATRIC GLIONEURAL TUMOR
}

Tania Mamdouhi ${ }^{1}$, Anshul Vagrecha ${ }^{2}$, Alan Johnson ${ }^{3}$, Carolyn Fein Levy ${ }^{2}$, Mark Atlas ${ }^{2}$, and Julie Krystal ${ }^{2}$

${ }^{1}$ Donald and Barbara Zucker School of Medicine at Hofstra/Northwell

${ }^{2}$ Steven and Alexandra Cohen Children's Medical Center

${ }^{3}$ Long Island Jewish Medical Center

May 31, 2021

\section{SUCCESSFUL USE OF CRUSHED FORMULATION OF DABRAFENIB AND TRAME- TINIB IN A PEDIATRIC GLIONEURAL TUMOR}

Tania Mamdouhi ${ }^{1}$, Anshul Vagrecha ${ }^{2}$, Alan A. Johnson ${ }^{1,3}$, Carolyn Fein Levy ${ }^{1,2}$, Mark Atlas ${ }^{1,2}$, Julie I. Krystal ${ }^{1,2}$

${ }^{1}$ Zucker School of Medicine at Hofstra/Northwell, Hempstead, NY

${ }^{2}$ Department of Pediatrics, Division of Hematology/Oncology and Cellular Therapy, Cohen Children's Medical Center, New Hyde Park, NY

${ }^{3}$ Department of Radiology, Division of Neuroradiology, Long Island Jewish Medical Center, New Hyde Park, NY

Corresponding Author : Julie Krystal. Cohen Children's Medical Center, 269- $0176^{\text {th }}$ Avenue, Suite 255, New Hyde Park, NY 11040. Jkrystal12@northwell.edu. Phone 718-470-3460. Fax 718-343-4642.

Tables: 0

Figures: 1

Supporting Information Files : 0

Short running title: Use of crushed formulation of dabrafenib and trametinib

Keywords : Dabrafenib, Trametinib, BRAF, crushed

Data Availability: The data that support the findings of this study are available from the corresponding author upon reasonable request.

TO THE EDITOR,

The combined use of dabrafenib and trametinib was approved by the U.S. Food and Drug Administration (FDA) for use in BRAF V600E mutated non-small cell lung cancer and melanoma in 2017 and 2018, respectively. Use of targeted therapy regimens in pediatric low-grade glioma have been under investigation. ${ }^{1}$ Dabrafenib is commercially available as a $50 \mathrm{mg}$ and $75 \mathrm{mg}$ capsule. As per the FDA, it should not be opened or crushed. ${ }^{2}$ Trametinib is distributed as $0.5 \mathrm{mg}$ and $2 \mathrm{mg}$ film-coated tablets to be swallowed whole. ${ }^{3}$ 
Phase 1and 2 of a study on dabrafenib use in pediatric cancers offered both the commercial capsule and an investigational suspension formulation of the drug for patients unable to tolerate capsules. ${ }^{1,4}$ Similarly, an oral suspension of dabrafenib and crushed administration of trametinib were used temporarily for patients unable to swallow. ${ }^{5}$ Suspension had a faster absorption and higher peak serum concentration, however similar exposure to the drug was seen when compared to capsular administration of dabrafenib. In this study, the bioavailability of the suspension was $85 \%$ of the capsule formulation. ${ }^{4}$

At our institution, we compounded a liquid suspension of trametinib and dabrafenib for those unable to swallow pills. In November 2019, a 17-month-old presented with right esotropia and was found to have a posterior fossa tumor with leptomeningeal extension into the brain stem and abnormal $\mathrm{T} 2$ prolongation in the upper cervical spine (Fig. 1A). She underwent a near total resection. Pathology demonstrated a high-grade glioneuronal tumor, with a BRAF V600E mutation and H3K27m wild-type. To avoid radiation, treatment was customized to target the BRAF V600E mutation. She was started on a compounded liquid suspension of dabrafenib ( $75 \mathrm{mg}$ once daily, $4.5 \mathrm{mg} / \mathrm{kg}$ ) and trametinib ( $0.5 \mathrm{mg}$ once daily, $0.03 \mathrm{mg} / \mathrm{kg})$ in December 2019 . The dose was adjusted for weight gain during clinical course. Imaging after two months and six months of treatment showed stable T2 prolongation and expansion in the upper cervical spine and stable medullary leptomeningeal disease. In October 2020, she was intubated for acute respiratory failure. Subsequently, she had a tracheostomy and gastrostomy tube was placed due to pharyngeal swallowing dysfunction. Due to swallowing dysfunction, crushed dabrafenib tablets and opened trametinib capsules were each administered through the gastrostomy tube in $5 \mathrm{ml}$ of water by the parents followed by a $10 \mathrm{ml}$ water flush. Follow-up imaging three months after showed significant improvement in both the leptomeningeal disease and size of expansile T2 prolongation in the cervical cord, consistent with treatment response (Fig. 1B). The patient continues the crushed dabrafenib and trametinib at this point.

In conclusion, a stable response with no tumor growth over 6 months using crushed dabrafenib and trametinib was observed in a case of pediatric glioneuronal tumor with BRAF V600E mutation. Although this is not an approved administration route, this patient's clear response to therapy demonstrates adequate absorption using this method. This outcome warrants further investigation into a broader study as crushing these tablets would facilitate their use for many patients with BRAF V600E mutated disease unable to swallow pills.

\section{CONFLICTS OF INTEREST}

Carolyn Fein Levy:

Owns stock in Pfizer.

\section{ACKNOWLEDGEMENTS}

None

\section{REFERENCES}

1. Hargrave, D. R.; Bouffet, E.; Tabori, U.; Broniscer, A.; Cohen, K. J.; Hansford, J. R.; Geoerger, B.; Hingorani, P.; Dunkel, I. J.; Russo, M. W.; Tseng, L.; Dasgupta, K.; Gasal, E.; Whitlock, J. A.; Kieran, M. W., Efficacy and Safety of Dabrafenib in Pediatric Patients with BRAF V600 Mutation-Positive Relapsed or Refractory Low-Grade Glioma: Results from a Phase I/IIa Study. Clinical Cancer Research 2019,25 (24), 7303-7311.

2. TAFINLAR (dabrafenib): Highlights of prescribing information. U.S. Food and Drug Administration: 2013.

3. MEKINIST (trametinib): Highlights of prescribing information. U.S. Food and Drug Administration: 2013.

4. Kieran, M. W.; Geoerger, B.; Dunkel, I. J.; Broniscer, A.; Hargrave, D.; Hingorani, P.; Aerts, I.; Bertozzi, A. I.; Cohen, K. J.; Hummel, T. R.; Shen, V.; Bouffet, E.; Pratilas, C. A.; Pearson, A. D. J.; Tseng, 
L.; Nebot, N.; Green, S.; Russo, M. W.; Whitlock, J. A., A Phase I and Pharmacokinetic Study of Oral Dabrafenib in Children and Adolescent Patients with Recurrent or Refractory BRAF V600 Mutation-Positive Solid Tumors. Clin Cancer Res 2019, 25 (24), 7294-7302.

5. Wang, J. R.; Zafereo, M. E.; Dadu, R.; Ferrarotto, R.; Busaidy, N. L.; Lu, C.; Ahmed, S.; GuleMonroe, M. K.; Williams, M. D.; Sturgis, E. M.; Goepfert, R. P.; Gross, N. D.; Lai, S. Y.; Gunn, G. B.; Phan, J.; Rosenthal, D. I.; Fuller, C. D.; Morrison, W. H.; Iyer, P.; Cabanillas, M. E., Complete Surgical Resection Following Neoadjuvant Dabrafenib Plus Trametinib in BRAFV600E-Mutated Anaplastic Thyroid Carcinoma. Thyroid 2019, 29 (8), 1036-1043.

Figure 1 A Axial T2 image prior to resection and treatment initiation demonstrates increased T2 signal and mild expansion of the cervical medullary junction worrisome for infiltrative tumor. B Axial T2 image 3 months after liquid suspension initiation shows no tumor progression with some improvement in treatment response.

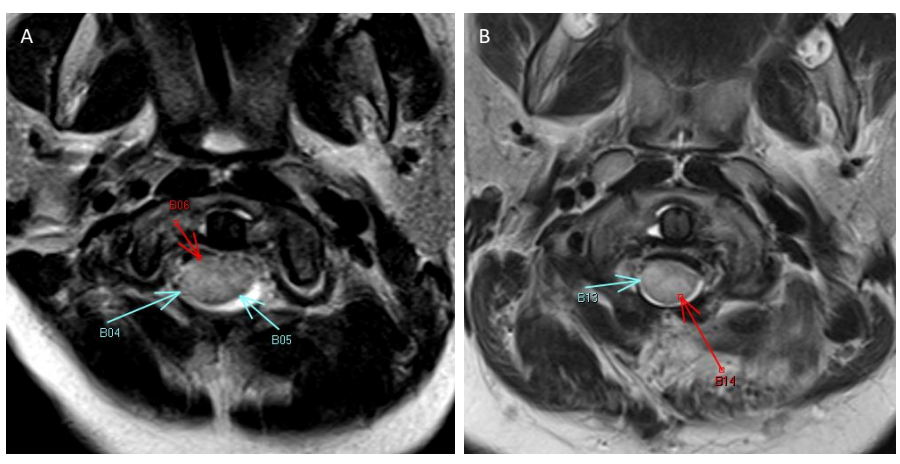

